\title{
Article \\ A Smart Capsule Equipped with Artificial Intelligence for Autonomous Delivery of Medical Material through Drones
}

\author{
Donatello Amicone ${ }^{1}$, Andrea Cannas ${ }^{1}$, Alberto Marci ${ }^{1}$ and Giuseppe Tortora ${ }^{1,2,3, *(D)}$ \\ 1 ABzero Srls, 56124 Pisa, Italy; damicone@abzero.it (D.A.); acannas@abzero.it (A.C.); amarci@abzero.it (A.M.) \\ 2 The BioRobotics Institute, Scuola Superiore Sant'Anna, 56127 Pisa, Italy \\ 3 Department of Excellence in Robotics \& AI, Scuola Superiore Sant'Anna, 56127 Pisa, Italy \\ * Correspondence: g.tortora@santannapisa.it
}

Citation: Amicone, D.; Cannas, A.;

Marci, A.; Tortora, G. A Smart

Capsule Equipped with Artificial

Intelligence for Autonomous Delivery of Medical Material through Drones.

Appl. Sci. 2021, 11, 7976.

https://doi.org/10.3390/

app11177976

Academic Editor: Paola Pellegrini

Received: 16 July 2021

Accepted: 26 August 2021

Published: 28 August 2021

Publisher's Note: MDPI stays neutral with regard to jurisdictional claims in published maps and institutional affiliations.

Copyright: (C) 2021 by the authors. Licensee MDPI, Basel, Switzerland. This article is an open access article distributed under the terms and conditions of the Creative Commons Attribution (CC BY) license (https:// creativecommons.org/licenses/by/ $4.0 /)$.
Featured Application: A Smart Capsule enabling medical staff to activate intuitively autonomous delivery mission of drones for transportation of medical materials.

Abstract: In the last few years, many examples of blood and medicine delivery drones have been demonstrated worldwide, which mainly rely on aeronautical experience that is not common in the medical world. Speaking about drone delivery, attention should focus on the most important thing: the transported lifesaving good. Traditional boxes that monitor temperature are not usually in real time, and are not suitable for drone transportation because they are heavy and bulky. This means that the biomedical characteristics of delivery are of primary importance. A Smart Capsule, equipped with artificial intelligence (AI), is the first system ever proposed to provide a fully autonomous drone delivery service for perishable and high-value medical products, integrating real-time quality monitoring and control. It consists in a smart casing that is able to guide any autonomous aerial vehicle attached to it, specifically designed for transporting blood, organs, tissues, test samples and drugs, among others. The system monitors the conditions of the product (e.g., temperature, agitation and humidity) and adjusts them when needed by exploiting, for instance, vibrations to maintain the required agitation, ensuring that goods are ready to be used as soon as they are delivered. The Smart Capsule also leverages external temperature to reduce energy uptake from the drone, thus improving the drone's battery life and flight range. The system replaces the need for specialized drivers and traditional road-bound transportation means, while guaranteeing compliance with all applicable safety regulations. A series of 16 experimental tests was performed to demonstrate the possibility of using the smart capsule to manage the flight and internal good delivery. Eighty-one missions were carried out for a total of $364 \mathrm{~min}$ of flight. The Smart Capsule greatly improves emergency response and efficiency of healthcare systems by reducing delivery times by up to $80 \%$ and costs by at least $28 \%$. The Smart Capsule and its enabling technology based on AI for drone deliveries are discussed in this paper. The aim of this work is to show the possibility of managing drone delivery with an AI-based device.

Keywords: medical delivery; drone delivery; telemedicine; autonomous robot; AI

\section{Introduction}

Nowadays the urgent transport of lifesaving goods, such as blood bags and samples, organs for transplants, health products, and tools, requires a specialized driver and an landbound vehicle (or helicopter where land means are not viable) [1]. This presents several flaws: they are affected by traffic conditions, prone to human mistakes, affected by pollutant and they are inefficient regarding current technologies. The whole request, preparation, and delivery process can prolong the waiting time for doctors and endanger a patient's life; meanwhile, the public and private healthcare systems have to face additional high costs for every minute lost. These problems worsen when it is necessary to reach 
remote mountain areas and islands in a short time. A Smart Capsule, which is flexible and modular, and equipped with artificial intelligence, is introduced in this paper. The Smart Capsule is able to guide whichever drone (or autonomous vehicle) it is attached to, with the final aim of replacing the presence of specialized drivers and traditional wheel-based means, regardless of the weather, and guaranteeing the conditions of its cargo according to current regulations. It also monitors the status (i.e., temperature and humidity) of the transported health goods, and modifies it when needed in order for it to be ready to be used as soon as it is delivered to the final destination. Artificial intelligence enables the possibility for the Smart Capsule to act as a supervisor during autonomous delivery missions in a broad range of medical applications.

Blood and blood components (i.e. plasma, red blood cells, white blood cells, and platelets) are vital healthcare resources used in a broad range of clinical services and are included in The World Health Organization's (WHO) model lists of essential medicines [2]. Transfusion is commonly used for supportive care in cardiovascular and transplant surgery, massive trauma, burn patient treatment, therapy for cancer, anemia, and pregnancy-related complications. Similarly, transplantation of organs is increasingly used as a medical treatment to save lives. Organ transplantation is necessary to treat end-stage organ failure and degenerative diseases. Access to sufficient, secure supplies of blood, blood products, and organs is an essential part of any strong health system.

However, blood components and organs have limited lifespans and need to be stored within correct temperature ranges and conditions, otherwise they become unusable. For instance, red cells must be maintained between $2{ }^{\circ} \mathrm{C}$ and $6{ }^{\circ} \mathrm{C}$, while the quality of plasma constituents is best maintained in a frozen state and platelet storage is optimal at room temperature $\left(20-24^{\circ} \mathrm{C}\right)$ with continuous agitation. Preservation is even more critical to organs, as the longer the delicate tissue is without blood circulation, the more damage occurs in cells. The right storage conditions shall thus also be maintained during transportation from blood banks and organ donors to where they are needed, and the transport shall happen quickly so as to not affect quality.

Currently, demand exceeds supply, and blood services find it difficult to make sufficient blood available, while also ensuring its quality and safety. Approximately 60,000 people in the EU and 80,000 in US are on a waiting list for a donor organ, with an average waiting time for a kidney transplant of 3.6 years and 1.5 years for a liver [3]. Organs and blood components cannot be manufactured, only donated, thus preserving them is a vital duty. In this context, drone delivery, eventually covering the "last mile" could be exploited to reduce the risk of organ damage due to long transportation times [4,5].

Despite the existence of very stringent legislation, it is generally estimated that more than $40 \%$ of blood transportation does not comply with the conditions imposed (e.g., deviation from temperature ranges), putting quality at risk, with negative consequences on costs (due to wastage or additional testing), as well as risks for patients. It can also increase the risk of bacterial proliferation in blood components during storage and may cause potentially life-threatening transfusion reactions, such as septic shock and even death. This situation is unacceptable since the need for blood and blood products is increasing every year and in many countries [6], particularly low- and middle-income countries.

Most urgent deliveries need to be addressed within $30 \mathrm{~min}$ from a call, but the traditional delivery service by road may be affected by heavy traffic, road closures, limited access to remote areas and unavailability of transporters [7]. Indeed, in several countries, it can take over $4 \mathrm{~h}$ to get blood from a blood bank to a patient, or from a patient to the closest lab for testing and diagnosis. The impact of poor or difficult road access on healthcare is well documented in both high- [8] and low-resourced [9] countries. Furthermore, with European directives imposing a reduction of small blood transformation facilities, blood courier services will have to greatly improve the quality of their services in order to maintain a high level of delivery efficiency. In the case of organs, these often travel for long distances and across country borders, even by plane or helicopter. 
In this context, unmanned aerial vehicles, such as drones, are becoming increasingly popular and have many potential applications in the biomedical sector, i.e., delivery of biological samples and medical supplies [10-13]. A drone delivery system can be used in any place, at any time and it will work in urban areas or remote locations. Drones have unique advantages, such as no traffic delays, low overhead costs, and the ability to go where there is no passable road. Drones are a potential way around these issues, but are only useful if they do not adversely affect the quality of transported products. Whether the quality of the product can be maintained throughout the duration of the flight remains a significant concern and a challenge [14].

The role of AI and deep learning in managing drones has been demonstrated to be very important at different levels in many fields [15-17]. This is particularly through when the delivery service is performed for medical purposes. However, these solutions are not focused on the most important thing, which is the good to be transported. Moreover, traditional boxes that monitor temperature are not usually in real time and are suitable for drone transportation because they are heavy and bulky.

In this paper, a Smart Capsule is proposed as a smart casing system that is able to automatically guide any unmanned aerial vehicle (drone hereafter), specifically designed for transporting blood and other medical products (e.g., organs, tissues, test samples, drugs) that need to be stored within strict conditions and delivered rapidly. The overall system includes dedicated electronics and an app to prove an intuitive delivery methodology that relies on drones managed by an AI-based device, the Smart Capsule.

\section{Materials and Methods}

The Smart Capsule is composed of a mechanical container in polyurethane, dedicated electronics, and the Spoke platform, composed of backend software, an app, and the electronic firmware of the Smart Capsule. In order to implement the basic needs of the Smart Capsule in terms of materials and manufacturing need, polyurethane material was selected after evaluating preliminary prototypes in carbon. The used materials are available off-the-shelf and were manufactured according to high-quality standards based on the design of the device. Some parts were manufactured out of metal for structural reasons (aluminum, steel) leaving room for weight reduction in future prototypes. The design takes into account the possibility of integrating a dedicated electronics board and antennas.

The mechanical interface of the drone has been manufactured out of aluminum to provide suitable mechanical resistance.

The Smart Capsule is a container with the following features:

- Autonomous flight control: no need for trained ground pilots;

- Fits all professional drones available on the market, as long as they comply with drone technical requirements (e.g., $10 \mathrm{~kg}$ load capacity, $60 \mathrm{~km} / \mathrm{h}$ minimum speed, water and wind proof) and is adaptable to the continuous evolution of drones thanks to a dedicated adjustable interface;

- GPS/GSM/3G/4G connectivity to control the drone anytime and anywhere. The connectivity allows to modify the flight according to flight authorization requirements. The connectivity also guarantees the recovery of the active control by drone operators in case of emergency. The connectivity is provided with a SIM7600 module (SIMCom, Shangai, China).

- Temperature and humidity sensors for real-time monitoring and control of storing conditions and quality of the medical freight, as prescribed by blood delivery regulations;

- Flexible and modular, able to adapt to any medical product (e.g., blood bags, organs, drugs, test samples).

The Smart Capsule is internally equipped with a certified UN3373 container equipped with temperature stabilizers that, together with the polyurethane envelope of the Smart Capsule, contribute to maintaining proper temperatures. The temperature stabilizers are cooled in order to reach a suitable temperature $\left(4^{\circ} \mathrm{C}, 22^{\circ} \mathrm{C},-25^{\circ} \mathrm{C}\right)$ before being inserted, together with the medical material, in the UN3373 container before every flight. 
Their action plus the thermal-insulated material of the Smart Capsule make temperature stabilization possible for about $3 \mathrm{~h}$, which is a more than suitable time for one delivery mission. The Smart Capsule is shown in Figure 1 together with a certified box for diagnostic blood delivery.

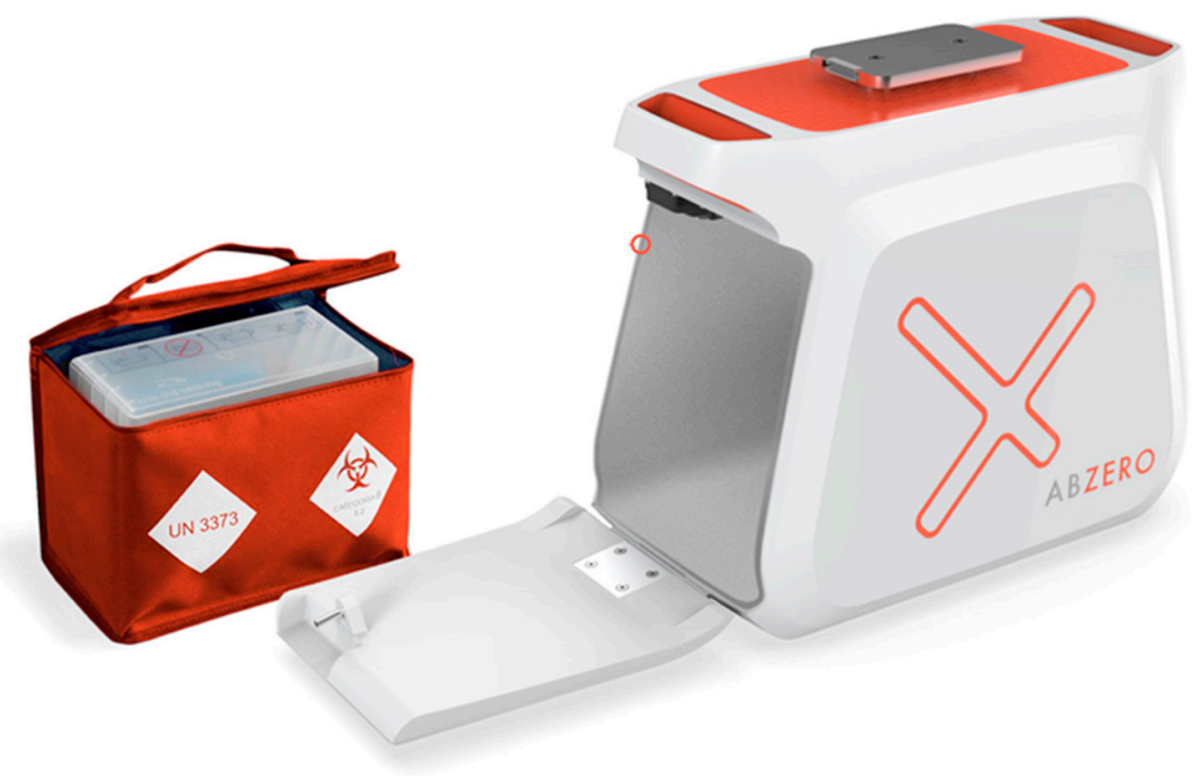

Figure 1. Smart Capsule 3D view with UN3373 container for blood sample delivery.

The Smart Capsule is designed for the delivery of blood and blood components, fulfilling stringent blood regulation, i.e., $4 \pm 2{ }^{\circ} \mathrm{C}$ for red cells, $25 \pm 2{ }^{\circ} \mathrm{C}$ for blood platelets, and $-25^{\circ} \mathrm{C}$ for frozen plasma.

Different capsules could meet the requirements of other medical product delivery operations with less stringent regulation, such as test samples, equipment, and other products (still with temperature needs of $4{ }^{\circ} \mathrm{C}$ ). The transportation of organs will be carried out by means of a specific internal certified container and by adjusting the temperature. The Smart Capsule is designed to be in full compliance with EU Directive 2002/98/EC prescribing quality and safety standards for the collection, testing, processing, storage, and distribution of human blood and blood components, and the smart capsule is UN3373 certified.

The temperature stabilizers are made of HDPE and contain a solution of water and paraffin mixtures, available off the shelf (provided by Rixlab, Gualdo Tadino, Italy). These modular stabilizers have been chosen as the best compromise for transportation and storage at a constant temperature of heat-sensitive products, such as blood components, diagnostic samples, drugs, etc. The temperature stabilizers are available in three versions, for transport at $+4{ }^{\circ} \mathrm{C},+22{ }^{\circ} \mathrm{C}$ and $-30{ }^{\circ} \mathrm{C}$, with the possibility of maintaining $-80{ }^{\circ} \mathrm{C}$ using dry ice. The possibility for the Smart Capsule to be integrated into different already-certified containers allows to cover the requirements for blood, medicine, and organs transportation by simply integrating suitable containers.

While these stabilizers correctly maintain the temperature in the correct ranges during traditional car transportation in only $60 \%$ of cases, the possibility of maintaining temperatures during drone flight has been demonstrated in this study.

In any case, the Smart Capsule will meet the good distribution practice (GDP) (both for the European Medicines Agency and the Food and Drugs Administration) in order to respect the minimum standards for ensuring the quality and integrity of medicines. These requirements are met by the Smart Capsule in this preliminary phase by integrating certified containers inside the main one. Although the integration of additional containers will be not convenient in the future due to their bulkiness and payload reduction, this 
solution was preferred in this phase in order to accelerate the use of the system in a real medical scenario.

The Smart Capsule mechanical structure was fabricated using CNC (computerized numerical control) techniques starting from a single part of polyurethane. Metal parts were also fabricated using traditional techniques. During series production, the costs of manufacturing will be drastically reduced through injection molding of plastic materials, improving both the mechanical and thermal characteristics of the device.

The Smart Capsule is designed to be compatible with any drone with the following features:

- Multicopter or VTOL (vertical take off and landing) drone

- Electric power

- $\quad$ LiPo or Li-ion batteries, plug and play charge

- IP53 protection from liquids minimum

- $\quad$ Resistant to $10-15 \mathrm{~m} / \mathrm{s}$ winds

- $\quad$ Cruise speed $10-15 \mathrm{~m} / \mathrm{s}$

- $10 \mathrm{~kg}$ payload, MTOW (maximum take off weight) $<25 \mathrm{~kg}$

- 20-30 or more energy autonomy, $12 \mathrm{~km}$ minimum distance one-way

- GNSS

- $\quad$ Pixahawk 2.1 optionally, including ADS-B

- Certified parachute

- Serial wired connection for the capsule

- Dimensions compatible with the Smart Capsule volume

The Smart Capsule is interfaced with the drone with a proprietary mechanical interface that allows electric connection between the drone flight controller and Smart Capsule electronics.

\subsection{Hardware Architecture}

The hardware/software system uses a microprocessor Broadcom based on low-energy ARM quad-core architecture energy consumption, which is then used by the Raspberry $\mathrm{Pi}^{\circledR}$ board. The serial communication and GPIO signals (general purpose input/output) were used for interacting with on-board sensors and for sending data. In the first phase of development, experimentation was performed through the use of a Breadboard to test the functionality of the microprocessor and check for any critical issues. The assembly allowed the starting of software development for specific board components, before getting to the final hardware prototype. The board was directly assembled using optimized instrumentation for surface mounting technology (SMT). The hardware board of the Smart Capsule included a $16 \times 2$-character LCD to display information from on-board sensors; a multicolored LED (red, green, blue) to inform the user about the system status; three wired sensors for measurement of the temperature in the different compartments of the Smart Capsule, including external and internal temperatures and humidity.

The hardware has the possibility of connecting additional sensors via Bluetooth Low Energy (BLE) to wirelessly connect temperature sensors; LTE-TDD/LTE-FDD/HSPA and GSM/GPRS/EDGE multi-band data connectivity for connection to the data network via different protocols was provided through a SIM7600 module by SIMCom. GNSS geolocation satellite receiver is also possible, with reception of GPS, BeiDou and GLONASS signals and guaranteeing correct positioning of the Smart Capsule. Management of an electric lock with an opening command and closing status was implemented. The electronics were equipped with power modules that were already compatible with the CEI EN 55022 standard, dedicated to information technology equipment, which specifies the limits in the frequency range of $9 \mathrm{kHz}-400 \mathrm{GHz}$. Furthermore, the radio sets are also certified according to the Radio Equipment Directives (2014/53/EU). The electronic components used complied with the RoHS (Restriction of Hazardous Substances Directive) which imposes restrictions on the use of certain dangerous substances in the construction of various types of electrical and electronic equipment.

The electronics were designed in a compact board embedding all the needed electronic hardware, allowing the easy integration inside the Smart Capsule. 


\subsection{Software Architecture and App}

Spoke is the proprietary software platform, specially designed for fulfilling the needs of medical staff for activating and monitoring the delivery. The Spoke platform includes the Spoke backend, the Spoke app, and Spoke AI (i.e., the software running on the Smart Capsule). The software was designed to interact with commercial flight control systems (Pixhawk, DJI) and is easy to use thanks to the Spoke app, which can be directly used by medical or non-expert staff to activate autonomous flights on pre-authorized routes. Thanks to the intuitive software, the whole delivery process can be managed directly by the healthcare facility, without the need of third-party couriers, thus improving efficiency and reducing costs and waiting times. It is enough to select the desired route from the app menu in order to be guided to activate the autonomous flight. In the following figure, the overall software architecture is shown, together with the app page for mission activation and live monitoring. The Spoke backend allows further monitoring of different missions performed by different systems running in parallel in a real working scenario.

The detailed software architecture of Spoke includes:

WEB PORTAL (i.e., Spoke backend): a tool for using the administration functions of the platform, monitoring and management of processes (back end user)

MOBILE APP (i.e., Spoke app): a tool for using functionalities of request, authorization and reception of transport in mobility (end user).

API LAYER: architectural component responsible for displaying services necessary for the implementation of user functions.

APPLICATION LAYER: application logics for the management of function systems in compliance with the designed logics.

PUSH NOTIFICATION: form for sending alerts and notes on mobile devices (end user).

INTEGRATION LAYER: module for the development of integration logics with external systems and the connectivity module.

CONNECTIVITY: sensor management logics (measurement acquisition and transmission of the same) and control of the Smart Capsule and the drone.

SMART CAPSULE (i.e., Spoke AI): the software running at the Smart Capsule level implementing AI, flight activation and supervision

DRONE: the software running on the commercial flight controller of the drone.

In addition to the software connection with the drone for commands related to flight and transport, the Spoke AI also manages the hardware components of the Smart Capsule, including sensors and, in the future, actuators that could be useful (i.e., cooling systems). The sensors integrated on Raspberry $\mathrm{Pi}^{\circledR}$ include internal temperature sensors and external sensors as well as humidity sensors. The hardware-software architecture guarantees that the sensors installed on board are constantly monitored and allows to detect anomalies in real time, both during the flight phase of drones and during the start-up of the activities related to the configuration phases.

The overall software architecture and the detail of the Spoke app are shown in Figure 2. 


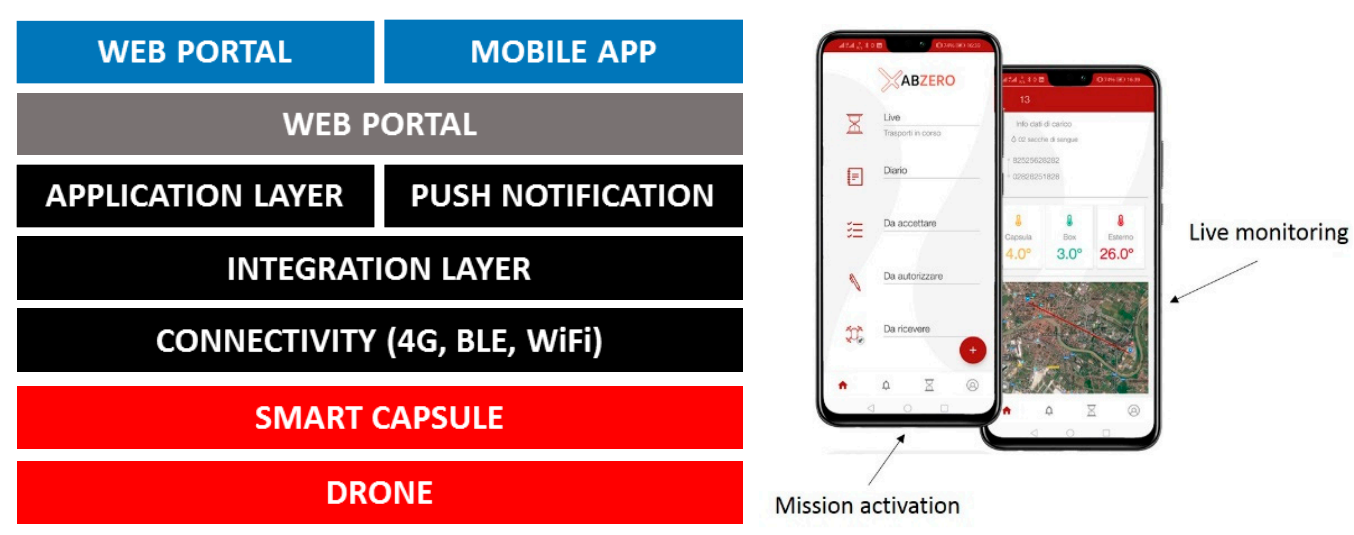

Figure 2. Overall software architecture (Left) and Spoke app (Right).

\subsection{Artificial Intelligence}

The Smart Capsule features a fully autonomous flight and control intelligence, thanks to which it can be operated by anyone (e.g., medical staff) with no need for a drone flight license, and which is in full compliance with applicable regulations to perform delivery missions in an autonomous and intuitive way, and in complete safety. The autonomous flight is activated and fully supervised by the Smart Capsule, in addition to drone machine tracking methodologies [18], whereas the flight is technically performed by a commercial flight controller integrated in the drone (e.g., Pixhawk open source flight controller). Thanks to the full flight and freight active control performed by its embedded artificial intelligence, the Smart Capsule is able to make autonomous decisions related to safety, such as stopping or pausing a flight, start a safe landing procedure, or change the mission during a flight. The device not only does away with specialized drivers and traditional road-bound transportation means, but also fully replaces the need for specialized drone pilots, while at the same time guaranteeing compliance with all applicable safety and medical delivery regulations. The Smart Capsule will always be an intelligent and unique device capable of intervening and correcting inaccurate trajectories in complete safety thanks to its own independent and redundant GNSS positioning system (mounted on the Smart Capsule electronics), in addition to the drone GNSS, and can benefit of the internal battery that allows the system to be located even in case of drone damage.

In the target application (delivery of highly valuable and perishable medical goods), the capsule allows to track the transported assets-which have the greatest value because they are aimed at saving lives-and is a device that can also be used alone and/or in collaboration with terrestrial drones, such as in a very broad Smart City and Industry 4.0 application cases. Proprietary algorithms have been developed based on machine learning approaches based on datasets that have been used. According to possible future developments, AI algorithms for visual imaging may be used (e.g., object recognition).

The role of AI is crucial. The on-board control intelligence is able to take over the control of the flying vehicles it is mounted on, or to make it redundant, thereby guaranteeing double insurance for successful mission completion, allowing the Smart Capsule to make autonomous decisions about distances, route changes, emergency maneuvers, and landings. AI makes it possible to meet the strict regulations of drone flight in urban scenarios by reducing the overall risk of operations through mandatory specific operation risk assessment, according to JARUS guidelines [19].

\section{Experimental Session}

The first phase was performed with the collaboration of the ABzero spin off company of Scuola Sant'Anna and the municipality of Pontedera (PI), Italy, by performing the first preliminary flight. From this collaboration, a prototype of the drone-transport cell was demonstrated, which was already certified for blood transport thanks to the internal UN3373 certified container (provided by Rixlab, Gualdo Tadino, Italy). 
The type of mission, with the related objectives and parameters verified, was carried out with the purpose of verifying the performance of the integrated system, consisting of one drone, the payload (Smart Capsule), and the Spoke platform, which are identified below for all configurations. It should be noted that this first phase of this analysis did not included the transport of blood and/or blood products as the goal was to validate the integrated system consisting of the elements mentioned above.

An optacopter drone with a 10-kg payload and the minimum requirements for interfacing the Smart Capsule (named ID8HSE) was selected for the experimental session.

The main aim of these tests was to demonstrate that the Smart Capsule could be used for activating and supervising the drone flight without interfering with any of the drone peripherals. Each mission that started from a takeoff waypoint and finished in a successful landing was considered successfully performed by the systems. In the future, the system will be intensively tested and perturbed in order to test the AI features of the Smart Capsule in depth. Figure 3 shows the system during a flight fully supervised by the Smart Capsule.

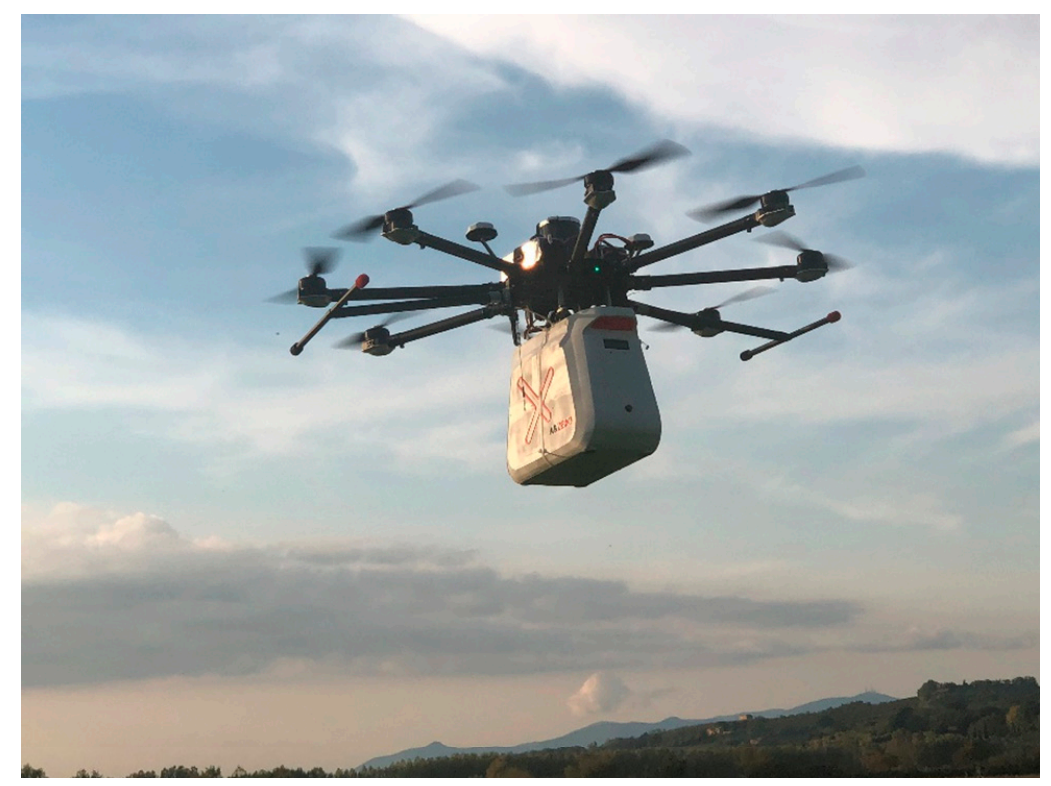

Figure 3. A flight fully supervised by the Smart Capsule AI during experimentation.

In the following, a series of categories and mission objective are reported.

1. Drone evaluation test-the test aims to verify the limitations provided by the manufacturer

2. Drone integration test-Smart Capsule (off): flight dynamics. The test aims to understand the flight dynamics of the drone on which the new payload, consisting of the Smart Capsule, is installed

3. Drone integration test-Smart Capsule (off): data-link loss. The test aims to understand the flight dynamics of the drone on which the new payload, constituted by the Smart Capsule, is installed, in the case of a data loss link.

4. Drone integration test-Smart Capsule (off): geo-fencing. The test aims to understand the behavior of the integrated system with geo-fencing activated

5. Drone integration test-Smart Capsule (off): collision avoidance. The test aims to understand the behavior of the integrated system in the presence of obstacles.

6. Drone integration test-Smart Capsule (off): waypoints accuracy. The test aims to verify the accuracy in the flight conduct of a waypoint previously set through the drone's proprietary app.

7. Drone integration test-Smart Capsule (on): flight dynamics. The test aims to understand the flight dynamics of the drone on which the new payload, constituted by the Smart Capsule, is installed. 
8. Drone integration test-Smart Capsule (on): data-link loss. The test aims to understand the flight dynamics of the drone on which the new payload, constituted by the Smart Capsule, is installed in the case of a data loss link.

9. Drone integration test-Smart Capsule (on): geo-fencing. The test aims to understand the behavior of the integrated system with geo-fencing activated.

10. Drone integration test-Smart Capsule (on): collision avoidance. The test aims to understand the behavior of the integrated system.

11. Drone integration test-Smart Capsule (on): waypoints accuracy. The test aims to verify the accuracy in the flight conduct of a waypoint previously set through the drone's proprietary app.

12. Drone integration test-Smart Capsule (on) - Spoke: flight dynamics. The test aims to understand the flight dynamics of the drone on which the new payload, constituted by the Smart Capsule, is installed.

13. Drone integration test-Smart Capsule (on)—Spoke: data-link loss. The test aims to understand the flight dynamics of the drone on which the new payload, constituted by the Smart Capsule, is installed in case of loss of data link.

14. Drone integration test-Smart Capsule (on)—Spoke: Geo-fencing. The test aims to understand the behavior of the integrated system with geo-fencing activated.

15. Drone integration test-Smart Capsule (on)—Spoke: collision avoidance. The test aims to understand the behavior of the integrated system.

16. Drone integration test-Smart Capsule (on)—Spoke: waypoints accuracy. The test aims to verify the accuracy in the flight conduct by waypoint previously set via the Spoke app with respect to the drone without the Smart Capsule.

The results of this first phase will be used to perform a further experimentation phase that will be carried out in more complex operational scenarios. Each mission was repeated under different environmental conditions, considering the need to check the behavior of the system even in the presence of wind.

Results

The Smart Capsule is a fully autonomous drone-based transportation system specifically designed for the transportation of blood, hemoderivatives, and other medical products (e.g., organs, tissues, swabs, test samples, drugs, etc.), which need to be maintained under strict conditions and rapidly delivered. The Smart Capsule features a full autonomous flight and control intelligence thanks to which it can be operated by anyone (e.g., medical staff or non-experts) with no need for a drone flight license, which is in full compliance with applicable regulations, to perform delivery missions in an autonomous and intuitive way, and with complete safety. Thanks to the active full flight and freight control performed by its embedded intelligence, the Smart Capsule, not only overcomes the limitations of traditional road-bound transportation by means of enabling medical staff to use a drone delivery service, but also fully replaces the need for specialized drone pilots, while at the same time guaranteeing compliance with all applicable safety and medical delivery regulations. The system can be fitted to any type of professional drone so as to be permanently compatible with state-of-the-art drone technology and future evolution. It implements real-time monitoring of freight and has wireless data transmission capabilities.

Eighty-one missions were carried out for a total of $364 \mathrm{~min}$ of flight, following the procedures identified in the experimental protocol. Missions were carried out in manual mode, to check some parameters and behaviors of the system, and in automatic mode, using both the commercial drone application and using planning through the Smart Capsule and the Spoke platform. For every test, data were acquired in order to qualitatively evaluate the behavior of the system under every condition in order to show that, after the integration of the Smart Capsule, no major changes were noticed in drone behavior.

The specific analysis of all drone parameters is outside of the scope of this paper. For the sake of clarity, a typical graph of vibrations along the axes is reported in the following image (Figure 4). Flight logs were saved in order to allow further reprocessing if necessary 
by the drone manufacturer and according to the requirements of the Italian Flight Authority (ENAC) for R\&D experimentation.

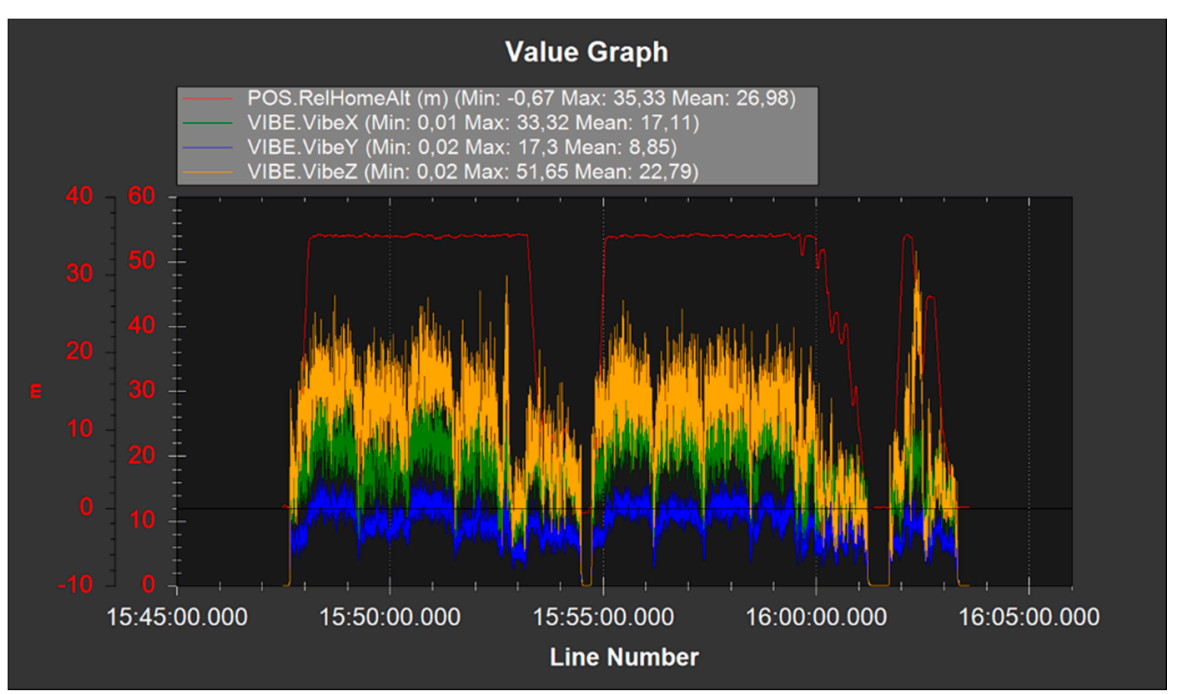

Figure 4. Vibration trends with the Smart Capsule mounted (note: comma is by used in this figure instead of dot for decimal separation).

Comparing this graph with those in which the Smart Capsule is not mounted on the drone, the same behavior can be observed, with peaks settling on the same values, so it is clear that the installation of the Smart Capsule does not cause a worsening of the flight conditions.

The experiments demonstrated that the Smart Capsule is able to activate and monitor flights without interfering with the basic control and safety system of the drone. A detail of a landing from the control station software is shown in Figure 5. Videos of the experimental flights are available online at www.youtube/abzerox.

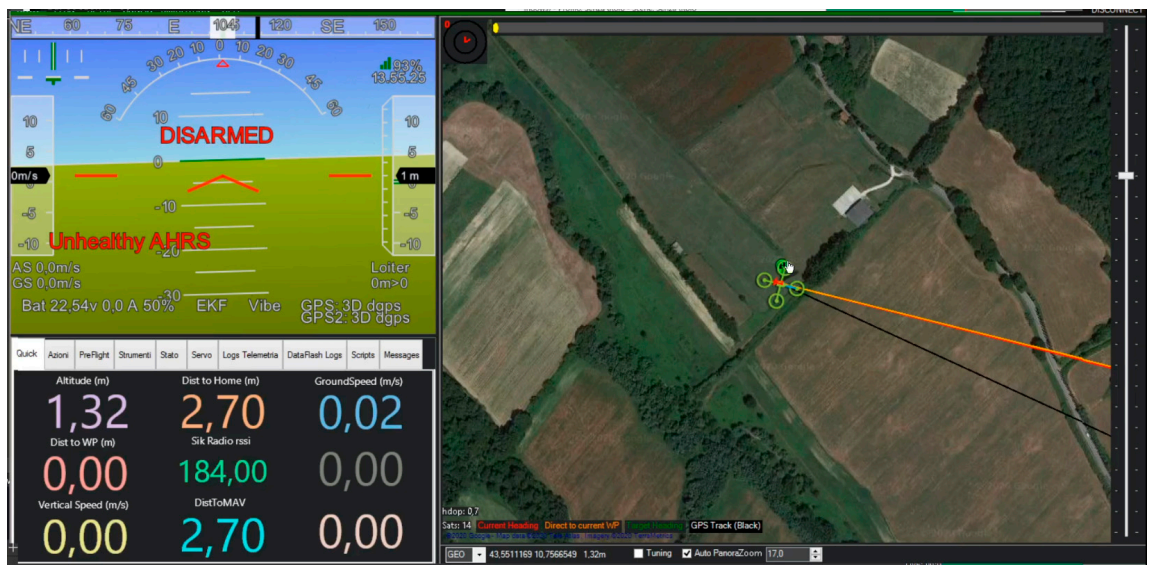

Figure 5. Drone data after a landing managed by Smart Capsule AI (note: comma is by used in this figure instead of dot for decimal separation).

\section{Discussion}

The scope of this work was the demonstration of possible applications of a Smart Capsule equipped with AI for the delivery of medical materials using drones to overcome current limitations in the regulatory framework and make autonomous delivery possible. Eighty-one missions were carried out for a total of $364 \mathrm{~min}$ of flight. The following types of missions were conducted:

Flights without Smart Capsule: 9—total flight time: $59 \mathrm{~min}$ 
Flights with Smart Capsule: 18 - total flight time: 62

Flights with Smart Capsule at maximum weight of $6 \mathrm{~kg}$ : 48 —-total flight time: 243 min Flight planned using Spoke app and software: 37-total flight time: $149 \mathrm{~min}$

The missions were carried out following an incremental process to verify the integration of the two subsystems, the drone plus the Smart Capsule. The first observation concerns the fact that no anomalies were found regarding the dynamics of the flight with the capsule mounted on board. The integrated system, therefore, maintained the safety levels required for this type of mission, even with the capsule loaded with a stable payload inside.

The vibrations recorded along the $\mathrm{X}, \mathrm{Y}$, and $\mathrm{Z}$ axes, without a capsule, had comparable values in terms of peaks with those with the capsule mounted (without and with a load of 2 to $6 \mathrm{~kg}$ ).

Additionally, with regard to the response to the commands, the same trends occurred, so it is believed that the installation of the capsule does not cause anomalies in terms of the flight dynamics. From the point of view of the behavior of the electronic system, no anomalies were found when the capsule was switched on. It is therefore believed that the integrated system maintained the safety levels required for this type of mission. The missions loaded with the Spoke application, developed by ABzero, were all successful; consequently, it is also believed in this case that the integrated system maintained the security levels required for this type of mission. The failsafe procedures, both in the case of RC loss or a low battery were always initialized at the right time, thus guaranteeing compatibility of the system with the assigned safety levels. The limits of flight geography were never exceeded.

From a system performance viewpoint, the following considerations must be made, which determine choices for the subsequent phases. The speed of the missions cannot be set to $5 \mathrm{~m} / \mathrm{s}$ as this increases the consumption of the batteries, even if there is a reduction in the accuracy of reaching waypoints; the presence of the Smart Capsule does not affect the overall system characteristics in terms of vibrations along the axes.

In comparison with other delivery systems based on drones [20,21], it is worth noting that the Smart Capsule represents an innovation since it introduces an additional intelligence level that can improve the overall safety of a system operating in an urban scenario. The Smart Capsule is, in fact, able to autonomously change a few parameters of a flight if negative events occur in order to guarantee the success of the mission, which can also continue using traditional delivery means after an emergency drone landing. Moreover, thanks to the integration of the already-certified containers inside the Smart Capsule, GDP were already met by the Smart Capsule, even in the experimental phase, which helps to reduce the time to market of the proposed solution.

The Smart Capsule implements a "total machine" concept, whereby a full active control on the drone it is coupled with the payload (goods transported) and the scope of the delivery mission is performed in a fully autonomous mode-that is, with no need for human operators nor pilots with a drone flight license-all while ensuring full compliance with applicable flying and medical regulations. Thanks to its fully autonomous flight and control intelligence (Spoke AI) and its complementary Spoke app, the whole delivery process can be intuitively managed directly by healthcare facility personnel, thereby fully removing the need for third party carriers and specialized drone pilots. The core control system of the drone is always active, as in traditional drone delivery systems, but is supervised by an intelligent system that, thanks to the redundant electronics and AI, reduces the risk of flying in complex urban environments.

With respect to the full range of commercial drone-based delivery systems and technologies, including the most advanced innovations that are currently under development worldwide, the Smart Capsule implements key groundbreaking innovations that soundly go beyond competing systems at the conceptual, technological and operational levels.

- At the conceptual level, it delivers a disruptive concept whereby the enabling technology is implemented at the capsule level, making it compatible with all professional 
drones. This strategic approach translates into a dramatic impact in terms its uptake and implementation potential

- At the technological level, it does not simply monitor, but actively controls the quality of the transported good on a real-time basis, and adjusting relevant parameters (temperature, agitation, humidity, etc.) in the case of drifting from the mandated ranges, thus making goods ready to be used as soon as they are delivered.

- At the operational level, its on-board intelligence and intuitive interface enables its uptake by medical staff.

\section{Conclusions}

In this work, a Smart Capsule with $\mathrm{AI}$ is been presented for the delivery of blood and blood components, together with a flight experimentation session to assess the benefits of the integration of an AI-based device as a supervisor for drone flights. Further studies will define the minimum training requirements needed for healthcare personnel to use the software through which they can download the recorded data or, possibly, intervene in the event of an accidental interruption of travel. The experimental session demonstrated that the Smart Capsule is able to activate and monitor flights without interfering with the basic controls and safety systems of the drone, representing a redundant device guaranteeing the safety of the operations.

Extensive tests on the Smart Capsule's temperature capability control will be performed in the future during the delivery of an experimental service in a real scenario. Analysis of the safety aspects of the system will be performed in the future in order to analyze the vulnerabilities of the drone system; in this activity, the cyber-security criticalities present from the viewpoints of the hardware and software, and to implement countermeasures to prevent the drone system from being tampered with by malicious users (i.e., hackers). The Smart Capsule will be also be analyzed from a thermal and mechanical viewpoint using FEM simulations and suitable crash tests will be performed for certification purposes. The capabilities of the Smart Capsule to react to undesired events in case of an emergency will further be improved by integrating AI inside the control loop in order to also implement suitable strategies for risk mitigation in case of emergency. In releasing the future service, labeling will be included for properly identifying the Smart Capsule in the medical environment.

In conclusion, this work demonstrated that the use of a Smart Capsule equipped with AI for enabling the flight of autonomous drone will represent a reliable solution for this kind of delivery to enable a new set of possibilities using drones managed by AI and redundant devices.

Author Contributions: The authors' contributions are reported as follows: technical implementation, D.A.; original concept, A.C. and G.T.; software implementation, D.A. and A.M.; validation, A.C. and G.T.; writing—original draft preparation, project management; funding acquisition. Principal Investigator, G.T. All authors have read and agreed to the published version of the manuscript.

Funding: This research was partially funded in the framework of the project Mini-lysis, JUMP (Joint Universities prograM for Poc), Ministero dello Sviluppo Economico (MISE), Italy.

Acknowledgments: The authors wish to thank Vania Di Francesco, Riccardo Petracca, Barbara Guidi for the support and ENAC for authorized this study. The authors also thanks Sergio Zamboni for his valuable support as business advisor.

Conflicts of Interest: The authors declare no conflict of interest.

\section{References}

1. Raptopoulos, A. No Roads? There's a Drone for That. TED Global 2013. Available online: https://www.ted.com/talks/andreas_ raptopoulos_no_roads_there_s_a_drone_for_that (accessed on 18 March 2018).

2. WHO Model Lists of Essential Medicines. Available online: https://www.who.int/groups/expert-committee-on-selection-anduse-of-essential-medicines / essential-medicines-lists (accessed on 10 July 2021). 
3. The Kidney Transplant Waitlist. Available online: https://www.kidney.org/atoz/content/transplant-waitlist (accessed on 10 July 2021).

4. Talaie, T.; Niederhaus, S.; Villalongas, E.; Scalea, J. Innovating organ delivery to improve access to care: Surgeon perspectives on the current system and future use of unmanned aircrafts. BMJ Innov. 2021, 7, 157-163. [CrossRef]

5. Eichleay, M.; Evens, E.; Stankevitz, K.; Parker, C. Using the unmanned aerial vehicle delivery decision tool to consider transporting medical supplies via Drone. Glob. Health Sci. Pract. 2019, 7, 500-506. [CrossRef] [PubMed]

6. Blood Safety and Availability. Available online: https://www.who.int/news-room/fact-sheets/detail/blood-safety-andavailability (accessed on 10 July 2021).

7. Claesson, A.; Fredman, D.; Svensson, L.; Ringh, M.; Hollenberg, J.; Nordberg, P.; Rosenqvist, M.; Djarv, T.; Österberg, S.; Lennartsson, J.; et al. Unmanned aerial vehicles (drones) in out-of-hospital-cardiac-arrest. Scand. J. Trauma Resusc. Emerg. Med. 2016, 24, 124. [CrossRef] [PubMed]

8. Arcury, T.A.; Gesler, W.M.; Preisser, J.S.; Sherman, J.; Spencer, J.; Perin, J. The Effects of Geography and Spatial Behavior on Health Care Utilization among the Residents of a Rural Region. Health Serv. Res. 2005, 40, 135-156. [CrossRef] [PubMed]

9. Bad Roads Hamper Deliveries of Needed Ebola Emergency Materials I The Liberian Observer. Available online: https://www. liberianobserver.com/news/bad-roads-hamper-deliveries-needed-ebola-emergency-materials (accessed on 10 July 2021).

10. Drone Delivery Becomes a Reality in Remote Pacific Islands. Available online: https://spectrum.ieee.org/the-human-os/ biomedical/devices / drone-delivery-becomes-a-reality-in-remote-pacific-islands (accessed on 10 July 2021).

11. Delivery Drones across Africa. Available online: https://spectrum.ieee.org/aerospace/aviation/zipline-expands-its-medicaldelivery-drones-across-east-africa (accessed on 10 July 2021).

12. When Drone Delivery Makes Sense. Available online: https://spectrum.iee.org/the-human-os/biomedical/devices/whendrone-delivery-makes-sense-when-youre-flying-lifesaving-blood-to-hospitals (accessed on 10 July 2021).

13. Drones Distribute Swarms of Sterile Mosquitoes to Stop Zika and Other Diseases. Available online: https://spectrum.ieee.org/ robotics/drones/drones-distribute-swarms-of-sterile-mosquitoes-to-stop-zika-and-other-diseases (accessed on 10 July 2021).

14. Amukele, T. Current State of Drones in Healthcare: Challenges and Opportunities. Laboratory Reflections. J. Appl. Lab. Med. 2019, 4, 296-298. [CrossRef] [PubMed]

15. Muñoz, G.; Barrado, C.; Çetin, E.; Salami, E. Deep Reinforcement Learning for Drone Delivery. Drones 2019, 3, 72. [CrossRef]

16. Dasu, T.; Kanza, Y.; Srivastava, D. Geofences in the Sky: Herding Drones with Blockchains and 5G. In Proceedings of the 26th ACM SIGSPATIAL International Conference on Advances in Geographic Information Systems, Seattle, WA, USA, 6-9 November 2018. [CrossRef]

17. Levine, S.; Pastor, P.; Krizhevsky, A.; Ibarz, J.; Quillen, D. Learning hand-eye coordination for robotic grasping with deep learning and large-scale data collection. Int. J. Robot. Res. 2017, 37, 421-436. [CrossRef]

18. Kangunde, V.; Jamisola, R.S., Jr.; Theophilus, E.K. A review on drones controlled in real-time. Int. J. Dyn. Control. 2021, 1-15. [CrossRef]

19. JAR-DEL-WG6-D.04. 2. JARUS Guidelines on SORA. Available online: http:/ /jarus-rpas.org/publications (accessed on 10 July 2021 ).

20. Ling, G.; Draghic, N. Aerial drones for blood delivery. Transfusion 2019, 59, 1608-1611. [CrossRef]

21. Balasingam, M. Drones in medicine-The rise of the machines. Int. J. Clin. Pract. 2017, 71, e12989. [CrossRef] 